\title{
Reflexión de la práctica: la profesionalización del docente
}

\author{
Ofelia Moreno Hernández, Irma Pérez Casillas y Leticia Martínez Pérez
}

\section{Resumen}

En este artículo se reflexiona sobre la tarea del docente. Esta tarea implica llevar a cabo una profesión integral y compleja, pues son los maestros quienes, como profesionales, fortalecen las capacidades intelectuales, académicas, sociales y de convivencia de los alumnos a su cargo. Es por ello que desarrollar la práctica es un proceso formativo que implica fomentar el deseo de los alumnos por mejorar en diversos ámbitos.

Las estrategias propuestas no son exclusivas para la docencia porque la finalidad es mejorar el desempeño profesional aun en otras áreas, ya que al aplicar las prácticas se construyen perspectivas que responden a los enfoques mediante el análisis del trabajo cotidiano y resolución constante de situaciones problemáticas. Para comprender las transformaciones y potenciar permanentemente el quehacer docente, es indispensable sistematizar una práctica reflexiva en un habitus social, ya que ésta es una actividad que se practica en un ámbito colectivo.

Con la finalidad de cumplir con la función docente correspondiente, se requiere de la preparación y la actualización que orienten el acompañamiento y favorezcan la movilización de saberes, por lo que resulta necesario facilitar a los alumnos normalistas estrategias que promuevan un proceso ordenado y sistemático. De esta manera, se analiza y valora su práctica docente con criterios validados mediante técnicas y herramientas adecuadas, así como con una buena reorientación para realizar una práctica, no sólo reflexiva, sino crítica.

Palabras clave: formación docente, intervención docente, práctica docente reflexiva-crítica.

\section{Reflecting on Practice: Professionalization of the Teacher}

\begin{abstract}
This article reflects on the task of the teacher. This task implies carrying out a comprehensive and complex profession, since teachers are the ones who, as professionals, strengthen the intellectual, academic, social and coexistence abilities of the students in their charge. For this reason, developing practice is a training process that implies fostering the desire of students to improve in various fields.

The proposed strategies are not exclusive to the teaching labor, because the main purpose is to improve professional performance even in other areas of expertise. Applying transformation of practical practices implies building perspectives that respond to approaches through the analysis of daily work and constant resolution of problematic situations. Undoubtedly, in order to understand the transformations and promote the permanent improvement of the teaching duty, it is essential to systematize a reflective practice into a social habitus, as it is a practice that takes place in a collective environment.

It is assumed that in order to fulfill the corresponding teaching duty, it is necessary to prepare and update the assistance that guides and favors the mobilization of knowledge. In this sense, it is necessary
\end{abstract}

Recepción: 21/11/2018. Aceptación: 20/05/2020. Dol: http://doi.org/10.22201/cuaieed.16076079e.2020.21.5.8 
to provide teacher-training students with strategies that promote an orderly and systematic process for analyzing and assessing the teaching practice, validated by means of specific criteria and other tools. In consequence, this will carry out a practice that is not only reflective, but also critical.

Keywords: teacher training, teacher intervention, critical-reflective teaching practice.

\section{Ofelia Moreno Hernández}

ofe102009benc@gmail.com orcid.org/0000-0002-8354-6257

Egresada de la Benemérita Escuela Normal de Coahuila, así como de la Universidad Pedagógica Nacional campus Saltillo, cuenta con Maestría en Educación Media y Universitaria. Actualmente se desempeña como docente de Escuela Normal, cuenta con experiencia en atención al trayecto de práctica docente, colabora como investigadora del Cuerpo Académico en formación, Modelos y procesos innovadores de la enseñanza-aprendizaje y la gestión educativa, participa en el área de tutorías, da seguimiento a los proyectos de movilidad académica de la institución y asesora de titulación de modalidad de portafolio docente.

\section{Irma Pérez Casillas}

casillasperez1995@gmail.com orcid.org/0000-0002-8819-3323

Egresada de la Benemérita Escuela Normal de Coahuila, del Instituto Universitario España campus Saltillo y la Universidad Santander campus Saltillo. Cuenta con las licenciaturas en Educación Tecnológica, Educación Primaria, maestría en Educación Media y Universitaria, y doctorado en Ciencias de la Educación. En la actualidad se desempeña como Subdirectora Académica de la Benemérita Escuela Normal de Coahuila, con características de liderazgo y planeación estratégica, dominio del Trayecto de Práctica Profesional en los Planes de Estudio 2012 y 2018, Tutoría Educativa y forma parte del Cuerpo Académico en Formación.

\section{Leticia Martínez Pérez}

letty master@hotmail.com

Maestra de Educación Primaria y de Educación Superior, egresada de la universidad de Tlaxcala con la especialidad de Lengua y Literatura españolas, cuenta con maestría en Educación y Desarrollo Docente de la universidad Ibero campus Saltillo, doctorado en Ciencias de la Educación de la universidad Santander campus Saltillo. Se ha desempeñado como coordinadora de la mesa técnica del sostenimiento federal, asesora de INEA del equipo técnico en la Ciudad de México, asesora pedagógica del programa de PIENso a nivel estatal, actualmente labora en la Benemérita Escuela Normal de Coahuila como docente, asesora de titulación, coordinadora de colegiado, y colabora en el CAEF, Cuerpo Académico en Formación. 


\section{Introducción}

Este artículo comienza por reconocer que la educación en la actualidad es objeto de múltiples transformaciones orientadas a mejorar la calidad y las necesidades de la sociedad. El maestro que hoy se incorpora al campo laboral requiere de un perfil específico como el que determina Carmona (2008), quien indica que el maestro debe ser capaz de desarrollar una dimensión reflexiva que le permita conjuntar formas de pensamiento racional e intuitivo, que conformen un proceso constructivo único. Por tanto, es un compromiso de los formadores de maestros el propiciar el desarrollo del pensamiento reflexivo y crítico.

Actualmente, la participación del docente se enfoca en desarrollar competencias mediante la reflexión crítica. Sin embargo, esto no se reconoce como una temática nueva porque, en realidad, está vigente desde la antigüedad, como determina Montecinos (2003), quien, después de analizar gran cantidad de literatura, llega a la conclusión de que el aprendizaje reflexivo se encuentra en el origen de las aportaciones de Platón y Aristóteles, promotores del diálogo como la clave de la reflexión y el pensamiento crítico.

En la formación de maestros es interesante adoptar la idea de Rodríguez (2014), quien conceptualiza que la práctica reflexiva del docente implica actitud sistemática de análisis y valoración de su quehacer para diseñar nuevas estrategias que puedan incidir de manera positiva en su enseñanza. Es también pertinente rescatar el saber docente $y$, a su vez, que esté enfocado en situaciones, acciones y problemáticas concretas que permitan establecer una relación entre su formación práctica y teórica. Este esquema considera lo que determina Montecinos (2003) al sugerir que las diversas tendencias propician que la profesión docente esté pasando desde una cultura del ejercicio individual al profesionalismo colectivo. Además, esta apertura trae consigo una mayor heterogeneidad en el alumnado, la diversificación de las demandas a los establecimientos educacionales, la necesidad de articular una educación continua a lo largo de la vida, y la complejización del conocimiento y del mercado laboral que exigen la capacidad de trabajar en equipo, y que son algunos de los factores que propician esta transformación. En este sentido, se sugieren estrategias y recomendaciones factibles en la formación de los nuevos docentes para consolidar las competencias profesionales y optimizar su desempeño.

\section{La práctica reflexiva en las escuelas}

En la actualidad, la educación proyecta una nueva visión de la docencia, así como de la gestión del conocimiento profesional. Los maestros son convocados a trabajar en equipo. Se les pide que en los consejos técnicos diseñen, implementen y evalúen proyectos de mejoramiento para sus centros educativos. A su vez, se les demanda que enseñen un currículo más exigente a un grupo más diverso de alumnos, que llegan a las aulas con intereses, motivaciones y experiencias de 
vida, frecuentemente, muy distintas a las expectativas de sus profesores. Ya no basta con enseñar lo que el marco curricular ha establecido, también hay que entregar evidencias de lo que los alumnos han aprendido, lo que se les enseñó y así como algunas relativas a la calidad del propio desempeño docente.

Sobre lo anterior, se han hecho estudios que permiten reconocer que es una temática vigente y de gran interés, como define Carmona (2008), quien propone utilizar la filosofía como instrumento potenciador del pensamiento crítico, entendida como actividad en su acepción socrática, y como investigación y búsqueda del sentido a través del diálogo, con la intención de potenciar la capacidad de reflexión, de autoevaluación y autocorrección, de respeto y de convivencia entre los participantes para fomentar su desarrollo integral.

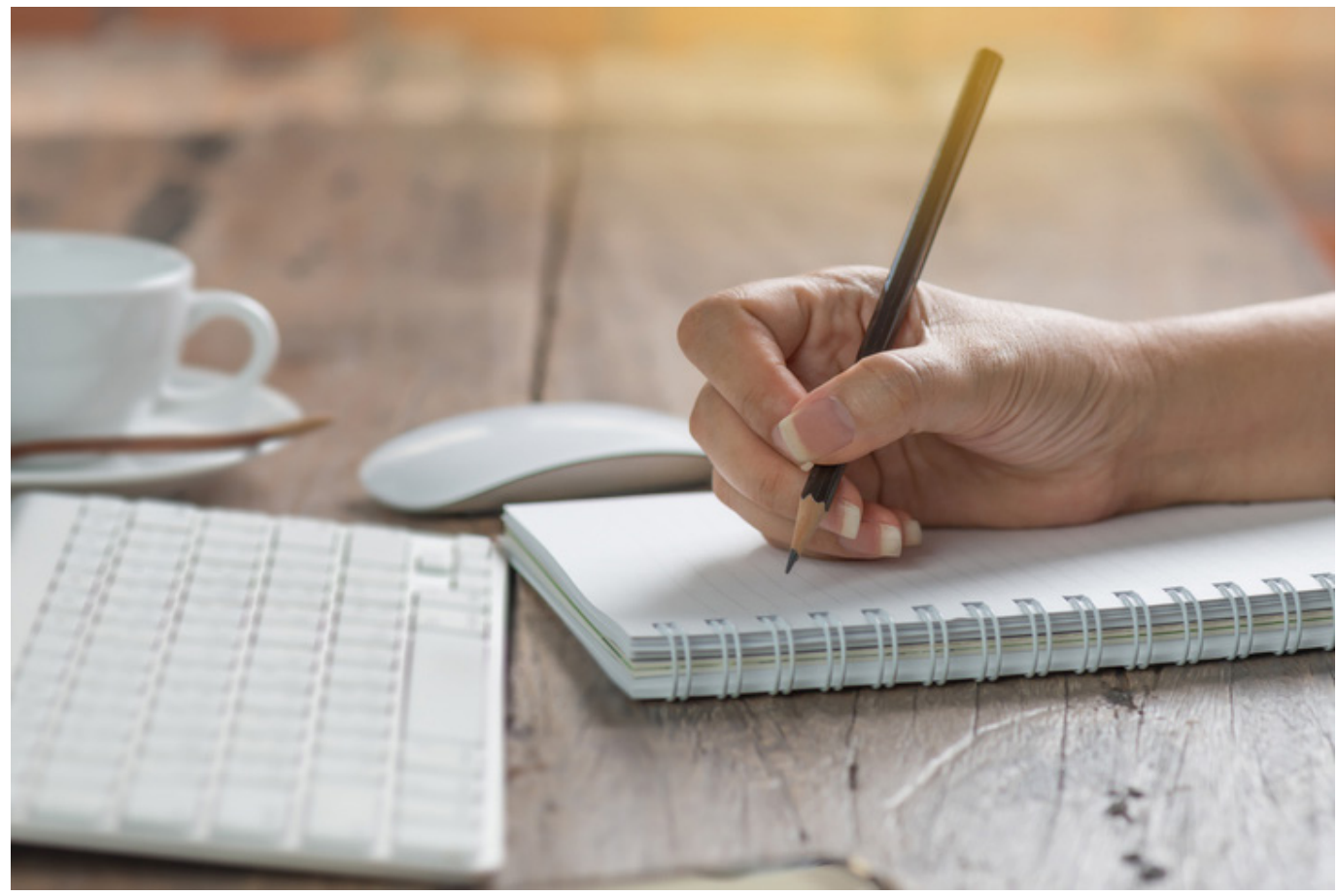

Al analizar las prácticas en las Escuelas Normales, se sintetiza que desarrollar competencias profesionales da la oportunidad de aprender de la propia práctica de forma habitual, y se prioriza la reflexión en y sobre la práctica en un hábito consciente, que se incorpora en la actividad cotidiana del profesional de la educación. Entre las bondades que genera, según Perrenoud (2010), esta metodología permite solucionar con éxito situaciones prácticas, favorece aprender de la propia práctica, promueve la construcción individual o grupal de propuestas para la mejora de la intervención docente mediante la reflexión metodológica, aporta profesionalidad al docente y transforma la práctica diaria en un proceso de investigación-acción. Asimismo, al desarrollar competencias profesionales se intenta comprender de forma crítica las situaciones con las que se enfrenta al abordar cuestiones y retos profesionales como objeto de investigación en pleno contexto real y no únicamente teórico. 
Por lo mencionado anteriormente, se reconoce que la práctica reflexiva y crítica propone analizar casos reales del escenario profesional, tomando en cuenta todos los elementos contextuales que permitan mejorar la formación práctica. De esta manera, se puede determinar como un ejercicio crítico, que implica incorporarse en un proceso de cuestionamiento, indagación, análisis y experimentación, de generación de nuevas estrategias de actuación, de reconceptualización de las creencias y teorías implícitas.

Como se puede apreciar en Perrenoud (2010), esta acción reflexiva y critica es fundamental para el desarrollo profesional y para lograr un aprendizaje permanente en la propia profesión, a través de sus intervenciones docentes, donde en el tiempo actual educar se ha vuelto cada vez más complejo.

Para fundamentar en este proceso de práctica reflexiva, Acuña (2015) apunta que el compromiso de las Escuelas Normales radica en vigilar los principios filosóficos del sistema educativo, promoviendo entre sus estudiantes la reflexión y la investigación como herramientas primarias en su formación y práctica profesional, buscando la relación oportuna con el entorno inmediato de la práctica escolar, a fin de mantener vínculos efectivos con el campo laboral y estar vigentes en la gran tarea que es formar docentes de calidad y con la intención de consolidar una formación profesional a los nuevos educadores.

Con esta propuesta se abre la posibilidad de cambiar el enfoque tradicional de la enseñanza-aprendizaje, para que sea congruente con el modelo educativo basado en competencias, donde el papel del maestro está centrado en atender los intereses de los alumnos, al buscar contextualizar el contenido, y donde también el papel del nuevo normalista deberá ser dinámico, investigador y propositivo. Esto último de acuerdo con el Rediseño Curricular de Escuelas Normales, que corresponde al acuerdo 14-07-18, en donde se establecen los planes y programas de estudio de la licenciaturas para la formación de maestros de educación básica. Éste incluye un planteamiento metodológico, globalizador y de aprendizaje en el servicio; la formación se basa en un modelo con la orientación del paradigma constructivista.

Para el éxito de la propuesta es preciso fundamentar que los planteamientos que se diseñen deben partir de las ideas previas de los estudiantes, y que mediante la situación didáctica se visualice la construcción de nuevos conceptos a raíz de la confrontación de ideas. Se sugiere que el proceso de enseñanza y aprendizaje sea significativo, que represente un reto para crear zonas de desarrollo próximo, pero, sobretodo, que genere y le permita al estudiante entrar en conflicto cognitivo. Hay que tener especial cuidado con que este esquema se desarrolle en un ambiente favorable y motivador, que le permita al estudiante estimular su autonomía y, por último, tomar en cuenta la metodología sugerida.

Al estudiar esta temática, se entiende que la reflexión es una postura intelectual metódica ante la práctica, que implica una intencionalidad por parte 
de quien la ejercita. Para lograrla es necesaria la curiosidad y la disciplina mental, pues estas características son hábitos reflexivos que no se improvisan, sino que son el resultado de un trabajo constante.

\section{Recomendaciones para la práctica reflexiva}

Durante la formación académica de los docentes se recomienda considerar el paradigma crítico, porque dentro de éste y según Carmona (2008) se trata de hacer énfasis en el desarrollo integral de la persona, es decir, de un tipo de ciudadano capaz de tomar en cuenta el punto de vista de otros, lo que le da oportunidad al estudiante de argumentar sus propuestas y sustentar sus decisiones de manera reflexiva y creativa. Cabe aclarar que es fundamental la solución conjunta de los problemas lo que genera que se propicie una interacción basada en el respeto mutuo, el razonamiento, la cooperación, la aportación constructiva y la coherencia ética. Para su aplicación y selección se propone la siguiente clasificación de estrategias que Brockbamk (2002) engloba por la participación y su uso, y que también depende de las cualidades del grupo donde se practique: estrategias socializadoras, talleres, tutoría grupal, seminarios. Los grupos de trabajos e implementación de innovaciones: las tríadas, taller reflexivo, método de proyectos, debates, elaboración de artículos, entrevista, aprendizaje basado en problemas, investigación y juego de roles. Y como estrategias individualizadoras: la lectura dirigida, la carpeta docente (diario reflexivo), el portafolio electrónico, tutoría individual, autobiografía y ensayo entre otras.

\section{Estrategias para la práctica reflexiva}

En cuanto al portafolio en físico o bien digital con las precisiones pertinentes, Rodríguez (2014) explica que el portafolio en físico facilita la organización de la información del trabajo docente, lo que genera mayor sistematicidad de la reflexión sobre su práctica educativa. Su contraparte digital suscita el uso de las tecnologías de información y comunicación (TIC) y es valorada como una herramienta novedosa y versátil.

El ensayo también es una estrategia factible para el análisis reflexivo y crítico. Se recomienda que el alumno revise un tema y que el material se complemente con argumentos relevantes según su posición correspondiente a la introducción, desarrollo y conclusión. Otra opción es el método de proyectos, que permite una mayor responsabilidad de su propio aprendizaje para rescatar, comprender y aplicar lo que aprenden los estudiantes.

El juego de roles también es una propuesta que promueve el desarrollo de pensamiento crítico en el aula. Rodríguez (2007) explica que puede ser una improvisación, que consiste en la representación espontánea o como una actuación, de acuerdo con un pequeño guion de una situación real o hipotética 
para mostrar un problema o información relevante. Su aplicación promueve conocimientos de forma significativa, pues se aprende a partir de la acción, así como el desarrollo de la empatía y la tolerancia, la socialización, la cooperación, la toma de conciencia, responsabilidad, y además favorece la capacidad de trabajo en grupo, la toma de decisiones y la resolución de problemas.

Asimismo, trabajar colaborativamente, como define Cano (2003), al aplicar la técnicas como lecturas, debates y análisis de casos, abre la posibilidad de incorporar programas de coaching en los que profesores con experiencia colaboran con los recién ingresados.

La videograbación es otra estrategia que regularmente se aplica en la reflexión de la prácticam porque permite autoobservarsey revisar detenidamente diferentes actuaciones para reconocer su imagenm y revelar fortalezas y áreas de oportunidad.

El uso de diarios es otra opción, ya que, si se desarrollan de forma sistemática y con las reorientaciones pertinentes, son el formato ideal para delinear la distancia entre la acción en el aula y la otra perspectiva, por lo que escribir es un buen método para el desarrollo reflexivo del docente. Al respecto, Zabalza (2011) categoriza los diarios para su análisis de la siguiente manera: como organizador estructural de la clase, descripción de las tareas, expresión de las características de los alumnos y de los propios profesores.

Al aplicar estrategias que propicien la práctica reflexiva y crítica, es recomendable tomar en cuenta los criterios de la tabla 1, que presenta Paul (2003) para mejores resultados.

\begin{tabular}{|c|c|}
\hline Todo razonamiento... & Criterios y procedimiento \\
\hline tiene un propósito & $\begin{array}{l}\text { Tómese el tiempo necesario para expresar su propósito con } \\
\text { claridad, que éstos sean realistas y significativos }\end{array}$ \\
\hline $\begin{array}{l}\text { es un intento de } \\
\text { solucionar un problema, } \\
\text { o explicar algo }\end{array}$ & $\begin{array}{c}\text { Tómese el tiempo necesario para expresar la pregunta en cuestión, } \\
\text { clarificar su alcance } \\
\text { Identifique si se trata de una opinión o si requiere que se razone } \\
\text { desde diversos puntos de vista }\end{array}$ \\
\hline $\begin{array}{l}\text { se fundamenta en } \\
\text { supuestos }\end{array}$ & $\begin{array}{l}\text { Identifique claramente los supuestos y determine si son } \\
\text { justificables }\end{array}$ \\
\hline $\begin{array}{l}\text { se hace desde una } \\
\text { perspectiva }\end{array}$ & $\begin{array}{l}\text { Identifique su punto de vista o perspectiva. Desde otros puntos de } \\
\text { vista e identifique sus fortalezas y sus debilidades }\end{array}$ \\
\hline $\begin{array}{l}\text { se fundamenta en datos, } \\
\text { información y evidencia }\end{array}$ & $\begin{array}{c}\text { Recopile suficiente información contraria a su posición, así como } \\
\text { información que lo apoye. Asegúrese que sea clara, precisa y } \\
\text { relevante a la pregunta en cuestión }\end{array}$ \\
\hline $\begin{array}{l}\text { se expresa mediante } \\
\text { conceptos e ideas }\end{array}$ & $\begin{array}{l}\text { Identifique los conceptos claves, explíquelos con claridad y } \\
\text { considere definiciones alternas; úselos con cuidado y precisión }\end{array}$ \\
\hline $\begin{array}{l}\text { permite llegar a } \\
\text { conclusiones }\end{array}$ & $\begin{array}{l}\text { Infiera sólo aquello que se desprenda de la evidencia, tanto } \\
\text { implicaciones como consecuencias positivas y negativas }\end{array}$ \\
\hline
\end{tabular}

Tabla 1. Principio o tipo de razonamiento y criterios aportados por Paul (2003). Fuente: elaboración propia, basado en Paul, 2003 


\section{Conclusión}

Con los planteamientos anteriores se determina que la reflexión se logra mediante un trabajo constante. No puede ser pensada como un proceso espontáneo (Rodríguez, 2014), si no que requiere una estructura y un andamiaje para promover una reflexión más profunda. Desde esta perspectiva, es conveniente contar con una guía que oriente y dé pautas para contribuir a que el profesor logre niveles más profundos de reflexión. Además, algunos de esos ejemplos pueden servir como "modelos" para que los profesores empiecen a incursionar en la escritura de su propio texto y, por último, el acompañamiento de un tutor para que compartan sus reflexiones, sus ideas y sus hallazgos.

Como experiencia personal, se sugiere seleccionar las estrategias que potencialicen las capacidades y habilidades de los estudiantes, además de requerir constante preparación y actualización que orienten la reflexión crítica, y acompañamiento metodológico a los estudiantes. Es por ello que resulta prioritario manejar estrategias que promuevan un proceso ordenado y sistemático, para analizar y valorar su intervención docente, con criterios y herramientas válidas, que eviten la subjetividad para realizar una práctica no sólo reflexiva sino crítica mediante técnicas y herramientas adecuadas para valorar su quehacer en el aula, y mejorar la innovación de su propio quehacer docente. Se concluye con la idea de que sólo la práctica reflexiva permitirá profesionalizar la docencia por las cualidades metodológicas y teóricas que implica su desarrollo e implementación en la formación de maestros.

\section{Referencias}

- Acuña, F. (2015). Retos de la formación docente ante las exigencias del mundo g/obal. Centro Regional de Educación Normal.

* Brockbamk, A. y McGill, I. (2002). Aprendizaje reflexivo en la educación superior. Morata.

* Carmona, G. (2008). Hacia una formación docente reflexiva y crítica. Fundamentos filosóficos. Revista de teoría y didáctica de las ciencias sociales, 13, 125-146. https:// www.redalyc.org/articulo.oa?id=65216719007

* Cano, E. (2003). La mentoría como metodología formativa. En Armengol Asparó C. y Gairín Sallán J. (coords.) Estrategias de formación para el cambio organizacional (pp. 147-155). Wolters Kluwer.

* Montecinos, C. (2003). Desarrollo profesional docente y aprendizaje colectivo. Psicoperspectivas. Revista de la Escuela de Psicología de la Facultad de Filosofía y Educación Pontificia de la Universidad Católica de Valparaíso, II, 105-128. https:/l www.psicoperspectivas.cl/index.php/psicoperspectivas/article/viewFile/6/6

- Paul. R. (2003) La mini-guía del pensamiento crítico. https://www.criticalthinking. org/resources/PDF/SP-ConceptsandTools.pdf 
* Perrenoud, P. (2010). Desarrollar la práctica reflexiva en el oficio de enseñar. Graó.

- Rodríguez, R (2014). El desarrollo de la práctica reflexiva sobre el quehacer docente, apoyada en el uso de un portafolio digital, en el marco de un programa de formación para académicos de la Universidad Centroamericana de Nicaragua [tesis de doctorado, Universitat de Barcelona]. Tesis doctorals en Xarxa. http://hdl.handle. net/10803/108035

* Rodríguez Cruz, R. L. (2007). Compendio de estrategias bajo enfoque por competencias. http://www.itesca.edu.mx/documentos/desarrollo academico/compendio de estrategias didacticas.pdf

* Zabalza, M. A. (2011). Diarios de clase. Un instrumento de investigación y desarrollo profesional. Narcea.

\section{Cómo CITAR ESTE ARTículo}

* Moreno Hernández, Ofelia, Pérez Casillas, Irma y Martínez Pérez, Leticia. (2020, septiembre-octubre). Reflexión de la práctica: la profesionalización del docente. Revista Digital Universitaria (RDU), 21(5). Dol: http://doi.org/10.22201/ cuaieed.16076079e.2020.21.5.8 